\title{
EL DISCURSO DE LA RSC EN LOS MEDIOS DE COMUNICACIÓN SOCIAL
}

\section{THE DISCOURSE OF CSR IN THE MEDIA}

Mónica Viñarás Abad: Prof. Dra. Facultad de Ciencias de la Comunicación. Universidad Antonio de Nebrija mvinaras@nebrija.es

\section{CURRÍCULUM VITAE}

Doctora por la Universidad Complutense de Madrid (UCM) con sobresaliente cum laude y Premio Extraordinario de Doctorado (2005/06). Licenciada en Publicidad y Relaciones Públicas por la misma Universidad. Ha completado su formación de postgrado con el Master en Comunicación de Instituciones Públicas y Políticas (ICIE, 1997) y el Master en Protocolo (UNED, 2006).

Inició su carrera como docente en la Universidad Europea Miguel de Cervantes (Valladolid) en 2006 y desde 2007 imparte clases en la Universidad Antonio de Nebrija; también colabora en distintas sesiones en el Master en Comunicación y Arte de la UCM.

Su área de investigación y docencia es la comunicación corporativa y las relaciones públicas, así como algunos de sus ámbitos más específicos: aplicación de las nuevas tecnologías, protocolo, patrocinio y mecenazgo, etc.

En el área de investigación, ha participado en distintos congresos internacionales con la presentación de comunicaciones. Ha publicado diferentes artículos en publicaciones científicas como Telos, Trípodos, Global Media Journal, etc. Así mismo, 
también ha colaborado con capítulos de libros como Blogalaxia y periodismo en la Red, La metamorfosis del espacio mediático, etc.

Como parte de su actividad docente e investigadora, ha realizado otras actividades como, por ejemplo, colaboraciones como evaluadora de artículos para revistas científicas, miembro de tribunal de tesis doctorales, dirección de proyectos fin de carrera y master, etc.

En el ámbito profesional ha trabajado en agencias de publicidad como JW Thompson, en departamentos de comunicación como el del Ayuntamiento de Alcobendas, y en la actualidad ejerce en el departamento de comunicación de una pyme. Trabajo que compagina con la docencia y la labor investigadora.

\section{RESUMEN}

La responsabilidad social corporativa es, poco a poco, objeto de contenido en los medios de comunicación por su práctica en empresa e instituciones. Lo que comenzó hace veinte años, hoy en día se ha convertido en parte de la cultura empresarial y filosofía corporativa en muchas organizaciones. Esta tendencia social, es recogida por los medios con escepticismo, interpretada en algunas ocasiones como una técnica de mejora de imagen y reputación y no como compromiso social. La comunicación social es la encargada de recoger estas prácticas y educar a la sociedad en esta tendente práctica empresarial.

\section{PALABRAS CLAVE}

RSC - Comunicación corporativa - Organizaciones - Medios de comunicación Compromiso 


\begin{abstract}
Corporate social responsibility is gradually taking part of the media due to its practice in companies and institutions. What began twenty years ago, nowadays has become part of the business culture and corporative philosophy in many organizations. This social trend is collected by the media with scepticism, interpreted in some occasions as a technique of image and reputation improvement and not as social commitment. Social communication is responsible for gathering these practices and educating society in this business practice tendency.
\end{abstract}

\title{
KEY WORDS
}

Corporate social responsibility - Corporate communication - Mass media

\section{ÍNDICE}

1. Introducción

2. Naturaleza y concepto de RSC

3. Comunicación y RSC

4. La RSC y los medios de comunicación social

5. Conclusiones: el futuro de una tendencia

6. Bibliografía 


\section{TEXTO:}

\section{Introducción}

"La empresa ya no es una isla, no puede aislarse del contexto en el que opera, de sus impactos sociales y medioambientales, porque la sociedad les demanda comportamientos sostenibles (...) La idea de la RSE (responsabilidad social empresarial) es de largo recorrido, ya que la empresa no se vuelve sostenible de la noche a la mañana. Tardará en impregnar el tejido empresarial español, pero lo importante es que el camino se recorra bien" (Jáuregui, R., 2008).

La responsabilidad social corporativa (a partir de ahora RSC) ó responsabilidad social empresarial (en esta investigación se utilizará RSC, pues se entiende que ésta incluye su práctica también en las empresas y por lo tanto también recoge esta actividad) es una práctica empresarial que refleja un cambio socioeconómico a nivel mundial. Para muchos, se trata de una moda que permite a las empresas ganarse la confianza de sus públicos, generando una reputación corporativa acorde con los valores de hoy en día. Para otros, la RSC no tiene vuelta atrás, el compromiso adquirido por las empresas en esta línea responde a las exigencias de la sociedad, y su ejercicio no es una opción para las empresas. El tiempo, el transcurso de los años, irá depurando estas prácticas, que como toda novedad, han tenido un momento de explosión en cuanto a la calidad y cantidad, pero que poco a poco irá consolidándose dentro de la estrategia corporativa, y no como una técnica aislada en el ejercicio de la responsabilidad. 


\section{Naturaleza y concepto de RSC}

Nuestro mundo demanda una nueva responsabilidad al hombre. Nos hemos enriquecido tanto de nuestro entorno, de la naturaleza, de las personas más desfavorecidas, que, por fin, parece que una nueva conciencia se ha expandido por todo el planeta: la responsabilidad para con todo y todos con los que compartimos este lugar en el universo.

La responsabilidad no es una elección, es un compromiso exigible y exigido. En nuestro mundo globalizado todos y cada uno tenemos nuestro papel, desde el hombre como individuo hasta las empresas y organizaciones como entes socialeseconómicos que son. La empresa se ha convertido en una institución social de primera magnitud al asumir nuevas responsabilidades hacia el entorno en que está inmersa y del que forma parte. “... estamos asistiendo (...) a una paulatina transformación de este mundo complejo, paradójico y continuamente cambiante que nos ha tocado vivir en torno a una globalización cultural, política, social y económica sin precedentes en la historia contemporánea. Una etapa en la que sus protagonistas más visibles: Estados/gobiernos, empresas, sociedad civil y opinión pública representada y legitimada en muchos casos a través de los medios de comunicación social- también se encuentran en estos momentos en una clara metamorfosis y reestructuración de sus funciones, responsabilidades y obligaciones" (González, 2007:47).

En la actualidad, los teóricos de la organización contemplan, con frecuencia, a las organizaciones como «sistemas». "Un sistema es un conjunto organizado de partes o subsistemas que interactúan. Cada sistema afecta a los demás, así como a la organización total“"(Grunig, 1984:56). La Teoría General de Sistemas defiende que "la realidad social presenta los caracteres de un sistema, para interpretar y explicar los 
fenómenos sociales a través de vínculos de interdependencia que los relacionan y que los constituyen un una totalidad" (Xifrá, 2003:18).

Este enfoque explica cómo la interacción de las empresas con su entorno, y los otros sistemas, tiene efectos en el sistema global, y en cada uno de ellos por separado. Es decir, las empresas no son entes aislados, es más, debido a la globalización y el poder de grandes grupos empresariales, su responsabilidad con el entorno es cada vez mayor. El entorno afecta a la empresa, y su desarrollo y estabilidad. Por lo tanto siguiendo este razonamiento, los cambios sociales afectan a las decisiones empresariales y su comportamiento con la sociedad. Su responsabilidad es proporcional a su papel en la sociedad.

Las empresas ya forman parte de las instituciones más poderosas del mundo, las 500 mayores empresas del mundo controlan el 25\% del volumen económico del planeta, y entre las 100 mayores potencias económicas mundiales, 50 de ellas son corporaciones empresariales (González, 2007:49).

Bien es sabido que los acontecimientos acaecidos en el panorama empresarial internacional, como el caso Enron ó Arthur Andersen, han provocado la desconfianza de inversores, accionistas, medios de comunicación, y opinión pública en general. Otros temas de interés social, como el cambio climático, el cuidado del medio ambiente, la igualdad entre las personas, el hambre, etc., son temas que aparecen en los medios de forma habitual. Cada vez son más los grupos de presión ó lobbies, que trabajan para conseguir mejoras sociales, presionando no sólo a legisladores y gobiernos, sino de forma indirecta también a las organizaciones.

En esta situación, las organizaciones están adoptando un papel que corresponde a las instituciones públicas, el de promover y desarrollar programas sociales, ya sea sobre medioambiente, acción social o la promoción del arte y la cultura. Las instituciones públicas, no tienen recursos para llevar a cabo todas estas iniciativas y las empresas asumen esta función "pública". Las empresas y organizaciones se han "hecho" 
responsables, desarrollando toda una serie de programas como si de organismos públicos se tratara, para dar respuesta a la demanda social de los grupos de interés con los que se relaciona. Así nace la RSC, para muchos, "puro marketing" en el sentido peyorativo (que no lo es) de explicar que el objetivo último es vender más, para otros, un cambio de mentalidad asociado a la situación social, económica, política y medioambiental que nos ha tocado vivir.

Para tratar de aclararlo y abordar qué es lo que se esconde detrás de este concepto, podemos recurrir a la definición dada en el Libro Verde aprobado en Julio de 2001 por la Comisión Europea, que define la RSC como la integración voluntaria por parte de las empresas de las preocupaciones sociales y medioambientales en el desarrollo de sus operaciones comerciales y sus relaciones con sus grupos de interés interlocutores; es decir, es el conjunto de prácticas empresariales que se derivan de los compromisos éticos que las organizaciones establecen con sus grupos de interés, así como, el reconocimiento e integración voluntaria de los mismos en sus operaciones, tanto en el ámbito social como medioambiental. (Comisión Europea, 2001:6-7) Es importante distinguir que es una decisión "voluntaria", que debe estar "integrada con las operaciones comerciales", y que se deriva de los "compromisos éticos que las organizaciones establecen con sus grupos de interés".

Entre las instituciones que han aparecido en los últimos años como respuesta e iniciativa a este movimiento, encontramos un concepto bastante similar al anterior, como el de Forética “La Responsabilidad Social de las Organizaciones es el fenómeno voluntario que busca conciliar el crecimiento y la competitividad, integrando al mismo tiempo el compromiso con el desarrollo social y la mejora del medioambiente". Sin embargo, otros, como la del Observatorio de la RSC, también entienden que el cumplimiento de las leyes, no voluntario por lo tanto, es una práctica de la RSC. "La Responsabilidad Social Corporativa (RSC) es la forma de conducir los negocios de las empresas que se caracteriza por tener en cuenta los 
impactos que todos los aspectos de sus actividades generan sobre sus clientes, empleados, accionistas, comunidades locales, medioambiente y sobre la sociedad en general. Ello implica el cumplimiento obligatorio de la legislación nacional e internacional en el ámbito social, laboral, medioambiental y de Derechos Humanos, así como cualquier otra acción voluntaria que la empresa quiera emprender para mejorar la calidad de vida de sus empleados, las comunidades en las que opera y de la sociedad en su conjunto" (Observatorio de la RSC, 2009).

Por lo tanto, existen posturas muy distantes para delimitar la responsabilidad de la empresa, desde Friedman, que entiende que la corporaciones tienen únicamente responsabilidad en el primer nivel, de manera que la empresa es socialmente responsable cuando esto incremente sus beneficios, no debiendo gastar dinero del consumidor y del accionista en problemas sociales (Friedman, 1970:17), hasta el "fenómeno voluntario" que hemos visto en los postulados anteriores.

La RSC es real, por obligación o por voluntariedad, las organizaciones están asumiendo prácticas impensables hace pocos años, que, por otra parte, conviven con la agresividad y competencia que caracteriza a estas organizaciones en sus estrategias comerciales.

Este cambio se refleja en los medios de comunicación social, que recogen en sus noticias temas relacionados con la RSC, incluso con secciones fijas para estas iniciativas. Una amplia representación de afiliados de APIE (Asociación de Periodistas de Información Económica) suscribe que "la Responsabilidad Social Corporativa es un compromiso que las empresas adquieren con la sociedad más allá de lo que marca la Ley" (Gil, 2007:77). 


\section{Comunicación y RSC}

Las RSC es una cuestión social, no económica, que intenta de alguna manera reconstruir la relación de la empresa con la sociedad y poner límites a determinadas acciones desarrolladas, sobre todo por empresas multinacionales que generan un impacto negativo sobre los derechos humanos y el medio ambiente en su ámbito de influencia. "Este nuevo escenario debe construirse con un enfoque multistakeholder y sobre una base de transparencia y confianza entre las partes que están involucradas en el proceso" (Vázquez, 2007:116).

El panorama económico-social actual obliga a las empresas a alcanzar resultados económicos positivos, a proteger y cuidar el medioambiente, a cumplir las leyes y obligaciones en temas sociales, y además, a hacerlo de manera voluntaria y proactiva más allá de lo que exige la Ley. Hace un siglo, las empresas -principalmente en EEUU- se dedicaban a ganar dinero a base de explotar a sus empleados en condiciones infrahumanas, con un único objetivo: ellas mismas. En este tiempo, las empresas no sólo cumplen las leyes, como es obligación, sino que además, promueven de manera voluntaria iniciativas para mejorar las condiciones de trabajo, insertar a grupos desfavorecidos, proteger su entorno, y promover acciones alejadas de sus objetivos económicos. Esto no quiere decir que todavía no se cometan injusticias, abusos y acciones reprobables, es un camino lento, muy competitivo y agresivo, y no hay que olvidar que sólo son veinte años de contemporánea RSC.

El primer paso está dado, el camino ha comenzado, y no hay vuelta atrás a esta nueva conciencia social, tal vez las gravísimas situaciones de crisis puedan hacer que sea más lento, pero el concepto de crecimiento y desarrollo ha cambiado.

La RSC debe ser comunicada, tal y como lo refleja el Libro Verde de la Unión Europea de 2001, sobre RSC, al afirmar que «ésta debe integrarse en sus relaciones con sus interlocutores», es decir, debe formar parte de lo que denominamos 
comunicación corporativa. Para Piñuel (1997:93), «la comunicación corporativa comprende formas de comunicación cuyos públicos destinatarios no se definen como consumidores de productos (...) sino como interlocutores sociales». Por lo tanto, la RSC debe integrarse en lo que se conoce como comunicación corporativa.

En este marco, las empresas asumen esta responsabilidad como uno de sus valores y principios corporativos, formando parte de la cultura de la empresa, como una seña de identidad. La identidad corporativa es «la esencia de la empresa» según Villafañe (1999:17) y está constituida por una serie de atributos que se van incorporando. La cultura corporativa incluye: los principios conceptuales en que se basarán los comportamientos expresivos de la organización hacia el entorno; los «valores» que comparten sus integrantes; y los «valores» sociales con los que se identifica. (Marín, 2008:80). Por lo tanto, la empresa asume la RSC como un rasgo de su personalidad, y así lo hace saber, por obligación, y por diferenciación. La RSC debe ser comunicada.

Para Van Riel (2005:26), la gestión de la comunicación ha de ser integral y no fragmentada. Por ello, «la comunicación corporativa es un instrumento de gestión por medio del cual toda forma de comunicación interna y externa, (...) para crear una base favorable para las relaciones con los públicos de los que la empresa depende». En este proceso comunicativo, la empresa transmite -simbólica y conductualmenteaquellos valores y rasgos que la caracterizan, y que forman la imagen, a través las percepciones y experiencias, en la mente de cada persona y grupo. “Este concepto de imagen intencional, la asunción de la RSC ocupa un lugar importante como atributo de imagen, ya que una característica primordial, de esa responsabilidad, es que se realiza de forma voluntaria, lo que influye positivamente en las percepciones de sus grupos de interés" (Martín, 2008:84).

La empresa, este nuevo ciudadano social, quiere transmitir esta nueva conciencia corporativa, no basta con comunicar los valores funcionales, hay que hablar, 
también, de valores sociales y, entre otros, de valores éticos y de responsabilidad social, según Francisco Martín, valores emocionales, como medio para establecer vínculos emocionales con los stakeholderes. Cierto que la responsabilidad social no debe ejercerse con el único objetivo de conseguir imagen, notoriedad, reputación, etc. Sin embargo, es necesario darla a conocer a los grupos de interés para que estos puedan valorar el compromiso de las empresas de ir más allá de una teoría de mínimos, a la hora de respetar la legislación vigente y cumplir sus expectativas y de la sociedad en su conjunto.

Por lo tanto, la RSC es eficaz, cuando forma parte de la cultura empresarial, y se comunica para transmitir este valor a los interlocutores y la opinión pública, a través de distintos canales y los medios de comunicación social.

Esta idea de que la responsabilidad fuera un rasgo de la personalidad de las empresas, no es tan novedoso como se nos hace ver. La RSC y paralelamente la reputación corporativa ocupan un lugar directivo en las empresas y organizaciones, desde hace apenas diez años, vinculadas a la gestión de la comunicación corporativa, en mayor o menor medida, pero repercutiendo en la imagen de la organización de una manera estratégica. Sin embargo, a principios del siglo $X X$, hace casi cien años, mucho antes de que el término RSC ocupara espacio en los medios de comunicación, y ningún director ostentara este cargo, la RSC ya se abordaba teórica y académicamente, desde un enfoque social, empresarial y comunicacional. Desde la fecha simbólica de 1923, Eduard Bernays abogaba por la práctica de unas relaciones públicas socialmente responsables, en 1980 lo sentenció en la Universidad de Boston, “Las relaciones públicas son la práctica social de la responsabilidad" (Grunig, 1984:47).

En 1984, James Grunig, el teórico contemporáneo más influyente de esta disciplina, defendía a estos profesionales como "gente que lucha constantemente por hacer que 
sus organizaciones sean responsables ante los públicos a los que influyen" (Grunig, 1984:41). Otros autores, como Cutlip y Center mantienen la persuasión como un efecto deseable de las relaciones públicas, pero añadieron una buena reputación, una actuación responsable y la comunicación bidireccional “Las relaciones públicas son el esfuerzo planificado para influir en la opinión pública a través de la buena reputación y de una actuación responsable, basados en una comunicación bidireccional" (Cutlip, 2001:34-38). En el ejercicio de la profesión, la RSC se convierte en medio y objetivo de una práctica excelente. También son los propios profesionales de las relaciones públicas defienden esta postura: "La empresa ha dejado de ser únicamente una entidad económica y se ha convertido en una entidad social" (Burson, 1997:224-227).

Las relaciones públicas nacen como profesión mucho antes de tener un corpus teórico. Sus inicios están en el periodismo al servicio de una organización y la propaganda política, pero en la madurez, paralelamente a un desarrollo teórico, el concepto de relaciones públicas excelentes va ligado a la transparencia, a una comunicación bidireccional que persigue un entendimiento mutuo entre organización y públicos estratégicos. Bernays recalcaba, constantemente y sinceramente, el papel de las relaciones públicas en la protección del público y el bienestar social (Grunig, pág. 98-99). Cambió el concepto de relaciones públicas de «informar al público» por el de cómo «debería comprenderse al público y cómo sus necesidades deberían ser tenidas en cuenta». (Bernays, 1990:804). Muchas empresas es lo que hacen, decirle al público lo que saben que el público aceptará, sin un cambio en su actitud y su comportamiento hacia las necesidades y demandas del público. Siguiendo un modelo asimétrico bidireccional, pues los efectos de la comunicación y el comportamiento de la organización son asimétricos para organización y público. En la actualidad, los investigadores están empezando a desarrollar las teóricas y las técnicas para un modelo simétrico, en parte, porque la investigación reciente sobre comunicación ha presentado serias dudas sobre el poder de la persuasión de masas. 
Dicha investigación ha sustituido la persuasión por la comprensión como el objetivo principal de la comunicación (Grunig, 1984:103). Es en los años 80 cuando comienza a utilizarse realmente este modelo con cierta continuidad y representatividad en la práctica profesional, aun dentro de su carácter minoritario (Arceo, 1988:70).

Según Harold Burson, uno de los fundadores de una de las agencias de relaciones públicas más importantes a nivel internacional, Burson-Marsteller, aboga por un profesional que conozca la opinión pública, no para persuadirla, sino para atender a sus necesidades. "El ejecutivo de relaciones públicas proporciona una evaluación cualitativa de las tendencias sociales. Ayuda a formular políticas que permitirán que una corporación se adapte a estas tendencias. Y comunica -tanto interna como externamente- los motivos de estas políticas [...] “Un objetivo obvio para el profesional de las relaciones públicas en el entorno corporativo es asegurarse de que las instituciones de negocios se comportan como servidores del pueblo "(Burson, 174: 224-227).

Se puede por tanto concluir, que las relaciones públicas ya postulaban el ejercicio de la responsabilidad social como parte de su trabajo, como interlocutores entre la organización y el público, buscando un equilibro entre los efectos del ejercicio del objeto social en la organización y el público, la sociedad, y el entorno en general. Entendían que la RSC debía formar parte de la filosofía corporativa, de manera estructural, estratégica y cultural, en la vanguardia de la excelencia empresarial.

En un nivel táctico, la RSC repercute en la imagen de la organización, en un tono más persuasivo, sin embargo, a nivel estratégico, repercuten en la reputación corporativa, la confianza en la organización y en el establecimiento de relaciones beneficiosas en ambos sentidos. 


\section{La RSC y los medios de comunicación social}

Los medios de comunicación, como transmisores de la realidad que percibe el individuo, y a la única que tiene acceso fuera de su entorno más próximo, tienen un papel fundamental en el conocimiento y percepción de la RSC. "Los medios de comunicación se han ido transformando en una nueva institución social que interactúa con otros actores sociales, y con individuos que no viven aislados, sino que mantienen todo tipo de interacciones con los diferentes agentes. (...)... se dedican a situar en el espacio público los discursos del resto de instituciones sociales a través de sus canales de comunicación: prensa, radio, televisión, Internet, etc." (González, 2007: 49).

Actualmente, el paradigma de RSE, RSC ó RS se encuentra en pleno debate conceptual y pragmático sobre su dimensión y alcance. Tratándose de un concepto nuevo, los medios son quiénes le van a dar contenido hacia la opinión pública, a través del propio espacio mediático. Distintos estudios reflejan el escaso tiempo y espacio que dedican los medios a este tema. Según el último informe de la Fundación Empresa y Sociedad titulado "La acción social de las empresas en la prensa escrita durante 2005", publicado a inicios de 2007, el número de noticias sobre acción social en la prensa escrita ha pasado de 643 en 2004 a 1.918 en 2005, casi el triple. Otros estudios, como el de Forética, la OCU o de la Fundación Avina, revelan que los ciudadanos en porcentajes superiores al $80 \%$ aún no perciben cambios significativos en las actitudes de las empresas ante la sociedad y desconocen el significado y al repercusión del a RSE. "Por lo tanto, la RSE aún o ha calado en las sociedad porque no se ha sabido transmitir ni el significad, ni el alcance, ni los diversos aspectos que la componen" (González, 2007: 54).

Según Marcos Suárez, las noticias de RSE aparecen en los periódicos económicos, y en los generalistas aparecen mezcladas en secciones como Sociedad, Medio Ambiente 
o Economía. En radio y televisión, apenas aparecen este tipo de noticias. Otro motivo es que los propios periodistas tienen poco espacio y tiempo para estas informaciones y por su parte en las empresas, hay pocos interlocutores válidos, falta de transparencia, poca calidad en la información, etc.

Poca receptividad por parte de los medios, información considerada de poco interés informativo por los periodistas, desconocimiento, falta de especialización del profesional del medio, escepticismo, prejuicios, se identifica con tácticas de relaciones públicas y/o marketing, confusión de términos y sensación de vacío de los mismos, dificultad para convertir las ideas y principios en noticias, pocos datos concretos y datos de evolución, mayor peso de las noticias presentadas por las ONG en detrimento de iniciativas concretas empresariales, reticencia a nombrar grupos empresariales y marcas como responsables de acciones que van más allá del negocio (como patrocinios, mecenazgo, investigaciones, etc.).

Otros autores son más optimistas en cuanto al trato de la RSC en los medios. "Ahora, las informaciones se centran más en la propia responsabilidad y menos en las marcas. Se nota un poco más de preocupación empresarial y bastante menos de autopublicidad y marketing. El efecto es que las redacciones han comenzado a realizar artículos genéricos sobre este tema en los que recogen los contenidos de muchas de estas notas de prensa" (Gil, 2007: 65). Según la Fundación ECODES, hay cierta "falta de madurez" de los medios a la hora de seguir la materia (...) se traduce en la tendencia a informar sobre acción social sólo a raíz de algún evento concreto organizado por otros, y no a partir del prurito personal del periodista (Fundación Entorno y Fundación Empresa y Sociedad, 2005:35).

Los periodistas están acostumbrados a recibir numerosas notas de prensa de las organizaciones, con información más o menos "objetiva". El ejercicio de la RSC constituye, en muchas ocasiones, el tema de estas notas de prensa. “Los periodistas 
tienen distintas opiniones sobre el objetivo de las mismas: desde una tendencia de Marketing y Comunicación, un nuevo paso en la reputación social corporativa, hasta una tendencia de Respuesta Social, los que entienden que se trata de una respuesta social, de una exigencia de los inversores, hartos de opacidad y sorpresas desagradables" (Gil, 2007: 71).

Uno de los requisitos que debe cumplir la RSC es el compromiso de transparencia. La RSC debe comunicarse, hacerse conocer a los grupos de interés, y la sociedad en general. Para ello se utilizan distintos soportes. Según una investigación del grupo Inforpress, a través de 50 entrevistas a empresas cotizadas, las herramientas más utilizadas para comunicar la RSC son las memorias de sostenibilidad, el gabinete de prensa, las acciones de relaciones públicas, etc.

“La comunicación es parte del proceso de implantación de la RSC, no obstante es necesario que ésta sea producto de una relación bidireccional previamente establecida con los grupos de interés, que le permita a la empresa marcarse unos objetivos y desarrollar políticas y programas acordes con los mismos. La comunicación de RSC debe entenderse como un sistema de rendición de cuentas que la empresa ejercita periódicamente y que va dirigido a sus grupos de interés, para que éstos cuenten con la información suficiente para evaluar la obtención de resultados en el ámbito de la RSC" (Vázquez, 2007:119).

Por lo tanto, las empresas están obligadas a comunicar su ejercicio de la RSC, con ética y transparencia. Los medios, son los encargados de hacer llegar a la opinión pública estas iniciativas, y formar e informar sobre esta nueva situación económica y social. Hay que pensar, que las publicaciones a las que antes se hacía referencia, como las memorias, llegan a grupos de interés muy reducidos y específicos, siendo los medios de comunicación los que a través de sus soportes conducen a la opinión pública sobre los temas en qué pensar. Como consecuencia de esta responsabilidad, 
el periodista tiene la tarea de distinguir entre acciones de RSC con un fondo social, y acciones de marketing, donde el compromiso de la empresa está supeditado al objetivo de imagen.

\section{Conclusiones: El futuro de una tendencia}

Sea o no voluntaria, la tendencia en los medios a tratar el tema de la RSC, en cualquiera de sus formatos, puede considerarse positivo. Significa que la audiencia, y la opinión pública en general, está expuesta a una noticia cuyo valor radica en un beneficio social, ya sea humano, medioambiental ó cultural. Cuando una empresa hace pública su RSC, además de obtener una serie de beneficios en términos de imagen, está adquiriendo un compromiso para el futuro, ya no puede dejar de ejercer la RSC: no puede dejar de cuidar el medioambiente, ni dejar de promover la igualdad entre las personas, ni ayudar a aquellas comunidades que lo necesitan, no se puede dejar de ser responsable. Si lo hiciera, su imagen se vería seriamente perjudicada, la relación con sus stakeholders se vería debilitada, perdería la confianza de sus públicos y en detrimento en su reputación. Hemos visto como el ejercicio excelente en la gestión requiere asumir la RSC como un valor de la empresa, como un rasgo de su personalidad, y un ejercicio continuo y sistemático. Todavía se puede pensar que todas las acciones de responsabilidad responden a este principio, y en muchas ocasiones el primer objetivo se fija en términos de imagen y reputación de la organización, y no como parte de la filosofía corporativa, y principio/valor. Aún así, tampoco supone un menoscabo de la acción en sí, una acción responsable que antes no se realizaba. Es un paso hacia delante.

“El hecho de hacer pública la RSC de una empresa se exponen a un tema principios éticos (...) por eso las empresas cambian de actitud y acaban creyéndose el mensaje, no como un fruto reactivo temporal sino que creemos que acaba convirtiéndose en parte de su cultura de empresas y es por eso que debemos seguir trabajando en la 
implicación de los principales actores sociales a fin de que sigamos avanzando hacia un cambio social que comporte el respeto de los derechos laborales en cualquier parte del mundo" (Vilá, 2007:140). Es una labor fundamental de los medios de comunicación la educación de este nuevo valor, una tendencia en la sociedad que nos alcanza a nivel individual y organizacional. A través de los medios es como aprendemos a conocer y distinguir estas acciones -fuera de la comunicación comercial y publicitaria- legitimándose en los medios. En ellos recae, como muchas otras veces, distinguir entre acciones puramente comerciales -“maquillaje”- y el reconocimiento a un esfuerzo por parte de la organización por dar respuesta a las demandas sociales. La RSC es un enfoque que implica al conjunto de la sociedad y que estamos hablando, por tanto, de cuestiones de indudable trascendencia que refuerzan el papel de las ciencias sociales y humanas en la comprensión de la cada vez más necesaria simbiosis entre sociedad y empresa. La tematización es el mecanismo de formación de la opinión pública en el seno de la sociedad postindustrial a través del temario de los medios de comunicación.

Los media constituyen hoy en día una nueva institución social, que están formando parte de grandes empresas comunicativas, con la responsabilidad que conlleva, a la que además se le suma la responsabilidad de transmitir los asuntos que más puedan interesar a la sociedad. Sin olvidar que los medios de comunicación son uno de los stakeholders, grupo estratégico o de interés para la empresa.

El periodista, a nivel individual, aunque siguiendo un línea editorial, tiene que distinguir entre el pseudo-acontecimiento o la nota de prensa que busca la cobertura de los medios que mejoren su imagen y reputación, frente a acciones reales de RSC, acciones o planes que busquen mejorar nuestro entorno, nuestro trabajo y en definitiva nuestra vida. Por lo tanto, el primer paso, es la formación del periodista en RSC, para poder distinguir estas acciones. Se ha comprobado que la tendencia de estos profesionales es a juzgar con escepticismo estas acciones, y cuestionar su naturaleza. Hecho muy razonable si tenemos en cuenta la proliferación de estas 
acciones, acometida por muchas empresas, más por seguir una moda, buscando la cobertura mediática, que como parte de la cultura. La tendencia a ejercer la RSC en las empresas, acabará por consolidarse como parte de su cultura, de una forma estratégica y sistemática.

Es en los años 90 cuando la RSC empieza ser asumida por las empresas de una forma estratégica y estructural, paralelamente a ser objeto de contenido en los medios. Muchos autores sitúan sus inicios a principios de siglo, en los movimientos feministas y las leyes laborales que prohibieron los primeros excesos de la sociedad industrial, sin embargo, no puede entenderse RSC propiamente dicha, pues nos estamos refiriendo a la empresa u organización privada como actor de la RSC. Tal vez, el primer caso de RSC tal y como hoy lo conocemos, promovida por un empresario, fue Rockefeller, cuando, creó la fundación que lleva su nombre y la posterior construcción de un hospital para personas sin recursos; su asesor en ese momento, Ivy Lee -pionero en la profesión de relaciones públicas-, entendió que no podía continuar prosperando a costa de los más necesitados, sin devolver y contribuir al bienestar del entorno donde sus empresas se enriquecían.

Hoy en día, la RSC en las organizaciones es una tendencia ya consolidada, en muchas, como parte de la cultura corporativa y generadora de confianza y reputación; otras todavía ven en ella una forma más de "crear imagen" a corto plazo. Los medios de comunicación legitiman en sus espacios estas acciones, formando a la opinión pública en el reconocimiento de esta labor y su exigencia en el futuro, mejorando la calidad de vida de toda la comunidad y el entorno. Éste es el reto. 


\section{Bibliografía}

ARCEO, José Luis: La eficacia de la responsabilidad social en las relaciones públicas de vanguardia: una revisión a los planteamientos de Grunig, Madrid, 1994, Revista Universitaria de Publicidad y Relaciones Públicas, 1 (2a época).

BERNAYS, Eduard: Radiografía de un sector, Barcelona, 1990, ESRP-PPU.

BURSON, Harold: "The public relations function in the socially responsile corporation" en MELVIN ANSHEN (ed.), Managing the Socially Responsible Corporation, Nueva York, 1974, Macmillan.

CUTLIP, Scott y otros: Relaciones públicas eficaces, Madrid, 1987, Prentice Hall.

FRIEDMAN, Milton: The social responsibility of business is to increase its profits, Nueva York, 1970, New York Times, 13 de septiembre.

GIL, Xavier: “Los periodistas económicos ante la responsabilidad social corporativa" en GALINDO, Ángel y FERNÁNDEZ, Beatriz (Coordinadores), Responsabilidad Social Corporativa y Medios de Comunicación, Salamanca, 2007, Publicaciones Universidad Pontificia de Salamanca, pp. 63-72.

GRUNIG, Scott y otros: Dirección de relaciones públicas, Madrid, 1984, Gestión2000.

GONZALEZ, Marcos: "RSE y medios de comunicación" en GALINDO, Ángel y FERNÁNDEZ, Beatriz (Coordinadores), Responsabilidad Social Corporativa y Medios de Comunicación, Salamanca, 2007, Publicaciones Universidad Pontificia de Salamanca, pp. 45-58. 
INFORPRESS: "La comunicación con el pequeño accionista y la responsabilidad social corporativa: Nuevas tendencias en la comunicación financiera" en IESE Business School y Asociación de Periodistas de Información Económica (APIE), Madrid, 2006, Inforpress.

JAÚREGUI, Ramón: Curso de Verano Forética en Expansión, 14 de septiembre, Madrid, 2008.

MARÍN, Francisco (2008): Responsabilidad social corporativa y comunicación, Madrid, Fragua.

PIÑUEL, José Luis (1997): Teoría de la comunicación y gestión de las organizaciones, Madrid, Síntesis.

VAN RIEL, Cees: Comunicación corporativa, Madrid, 2005, Pearson/Prentice Hall.

VILA, Xavier: “El trabajo de Intermón Oxfam con medios de comunicación en temas de responsabilidad social corporativa" en GALINDO, Ángel y FERNÁNDEZ, Beatriz (Coordinadores), Responsabilidad Social Corporativa y Medios de Comunicación, Salamanca, 2007, Publicaciones Universidad Pontificia de Salamanca, pp. 135-141.

VILLAFAÑE, Justo (1999): La gestión profesional de la imagen corporativa, Madrid, Pirámide.

VÁZQUEZ, Orencio: "La información en torno a la responsabilidad social corporativa" en GALINDO, Ángel y FERNÁNDEZ, Beatriz (Coordinadores), 
Responsabilidad Social Corporativa y Medios de Comunicación, Salamanca, 2007, Publicaciones Universidad Pontificia de Salamanca, pp. 111-121.

XIFRÁ, Jordi: Teoría y estructura de las relaciones públicas, Madrid, 2003, McGrawHill.

FUNDACIÓN ENTORNO y FUNDACIÓN EMPRESA Y DESARROLLO: Desafíos de la comunicación de la Responsabilidad Social Corporativa y el Desarrollo Social en los medios, Madrid, 2005, Fundación Entorno.

http://www.accionsostenible.org

http:/ /www.analisiseinvestigacion.com/

http://www.empresaresponsable.com/

http://www.foretica.es/

http://www.institutointangibles.com/

http://www.isrcer.org/

http:/ /www.observatoriorsc.org/

http:/ /www.reputacioncorporativa.org/

http://www.serresponsable.es/index.php 